\title{
DIVERTÍCULO URETRAL TRAS URETROPLASTIA DE SUSTITUCIÓN. A PROPÓSITO DE SEIS CASOS
}

José Luis Lozano Ortega y Carlos Pertusa Peña.

Servicio de Urología. Hospital de Cruces. Baracaldo. Vizcaya. España.

Resumen.- OBJETIVO: Mostrar la patogenia, diagnóstico y posibilidades de tratamiento de los divertículos uretrales que pueden producirse tras realizar una uretroplastia de sustitución. Comentamos los seis casos que hemos recogido en una serie de 34 pacientes y las técnicas que pueden ser útiles para tratar de evitar esta complicación.

MÉTODOS: Sobre un total de 34 uretroplastias, 20 con colgajo pediculado y 14 con injerto de mucosa bucal hemos recogido seis casos de formación de un divertículo o saculación uretral. De ellos cinco han sido intervenidos y en un caso optamos por la abstención dado que no presentaba síntomas.
RESULTADOS: Cuatro pacientes permanecen asintomáticos en la actualidad tras el tratamiento. Los dos restantes están pendientes de cierre uretral en un segundo tiempo.

CONCLUSIONES: La aparición de pseudodivertículos uretrales tras cirugía de sustitución uretral por estenosis no es una complicación infrecuente. Existen técnicas que buscan reforzar la resistencia de ésta zona de la uretra debilitada y que es importante conocer en la práctica de esta cirugía reconstructiva.

Palabras clave: Divertículo uretral. Uretroplastia.

Summary.- OBJECTIVES: To expose the pathogenesis, diagnosis and therapeutic options for urethral diverticula appearing after substitution urethroplasty.

METHODS: Over a total of 34 urethroplasties, 20 with pediculated flap and 14 with buccal mucosa, we collected six cases of urethral diverticula/sacculation development. Five of them underwent surgery, and in one case without symptoms we opted for therapeutic abstention.

RESULTS: Currently four patients remain asymptomatic after treatment. The other two are waiting for a secondtime urethral closure.

CONCLUSIONS: The development of urethral pseudodiverticula secondary to stenosis after urethral substitution surgery is not an infrequent complication. For the practice of reconstructive surgery it is important to know that there are techniques to reinforce the resistance of this weakened area of the urethra. 
Keywords: Urethral diverticulum. Urethroplasty.

\section{INTRODUCCIÓN}

El tratamiento con técnicas abiertas de la estenosis de uretra incluye el uso de injertos libres y colgajos pediculados para aquellos casos en que la situación de la estenosis o su longitud no hacen aconsejable una cirugía de exéresis con anastómosis término terminal.

La elección de la técnica más adecuada y una cuidadosa ejecución de la misma, disminuyen en buena medida la tasa de complicaciones pero no evitan que se produzcan, como se deriva de cualquier acto quirúrgico y que, a la hora de evaluar los resultados, tendremos que incluir como "fallos", "fracasos" o "malos resultados". En el caso de la cirugía uretral las complicaciones más habituales, que afectan directamente a la neouretra van a ser, hematomas, fístulas, reestenosis parcial o total, necrosis, divertículos e infecciones. Su resolución a veces es sencilla, en ocasiones muy compleja y siempre hay que dejar un espacio al tratamiento conservador, es decir, no intervenir, si con ello también conseguimos el bienestar del paciente.

Presentamos una serie de seis divertículos secundarios a cirugía uretral de sustitución, su diagnóstico, evolución, síntomas y tratamiento.

\section{MATERIAL Y MÉTODOS}

Seleccionamos 34 pacientes, intervenidos entre 2002 y 2006 , en los que se han realizado uretroplastias para ampliar el calibre uretral. De ellos, 20 corresponden a colgajos pediculados y 14 son injertos con mucosa bucal. Los criterios de selección han sido incluir aquellos pacientes en los que se ha llevado a cabo una de las técnicas citadas, y cuyo seguimiento haya sido posible de forma periódica. Se han excluido otros tipos de uretroplastia y aquellos casos en que faltan datos de la evolución.

Hemos detectado seis divertículos uretrales, de los que cuatro se localizan en uretra peneana y dos en uretra bulbar. En el primer caso se había llevado a cabo una sustitución con colgajo pediculado de mucosa prepucial y en el segundo un injerto con mucosa bucal (Tabla I).

TABLA I.

\begin{tabular}{|c|l|c|l|l|l|}
\hline Edad & Localización & Longitud $(\mathbf{c m})$ & Técnica & Síntomas & Tratamiento \\
\hline 67 & Peneana & 4 & C.M.P. & $\mathrm{Si}$ & Puesta plano \\
\hline 75 & Penoescrotal & 3 & C.M.P. & No & No \\
\hline 71 & Penoescrotal & 5 & C.M.P. & $\mathrm{Si}$ & Reconstrucción \\
\hline 40 & Bulbar & 3 & M.B. & $\mathrm{Si}$ & Un tiempo \\
\hline 50 & Peneana & 3 & C.M.P. & $\mathrm{Si}$ & Puesta plano \\
\hline 36 & Bulbar & 4 & M.B. & $\mathrm{Si}$ & T-T Ampl.. \\
\hline
\end{tabular}


Cinco pacientes manifestaron síntomas irritativos u obstructivos, siendo el que apareció con mayor frecuencia el goteo persistente tras finalizar la micción. Tambien refirieron hematuria, escozor miccional, dolor en la zona del divertículo y disminución en la proyección del chorro miciccional. En un caso el paciente se encontraba cómodo y carente de síntomas.

Para el diagnóstico, cuando aparecieron los síntomas, utilizamos medición del flujo miccional, uretrocistografía combinada miccional y retrógrada y uretroscopia.

El tratamiento se llevó a cabo valorando los síntomas junto a los hallazgos radiológicos, endoscópicos $y$, algo que consideramos de mucha importancia, como es el aspecto de la mucosa durante el acto quirúrgico. De este modo realizamos una uretrotomía interna por una estenosis anular del extremo distal del injerto, dos puestas a plano o marsupialización del divertículo debido al aspecto inflamatorio del epitelio uretral, una reconstrucción en un tiempo mediante exéresis de mucosa sobrante y cierre uretral en varios planos, una uretroplastia término terminal ampliada con mucosa bucal en situación dorsal y por último decidimos no actuar en el caso del paciente asintomático.

\section{RESULTADOS}

En el momento actual, de los seis pacientes, cuatro se encuentran libres de síntomas y sin datos de recidiva. Los dos restantes corresponden a aquellos en que realizamos una puesta a plano del divertículo:

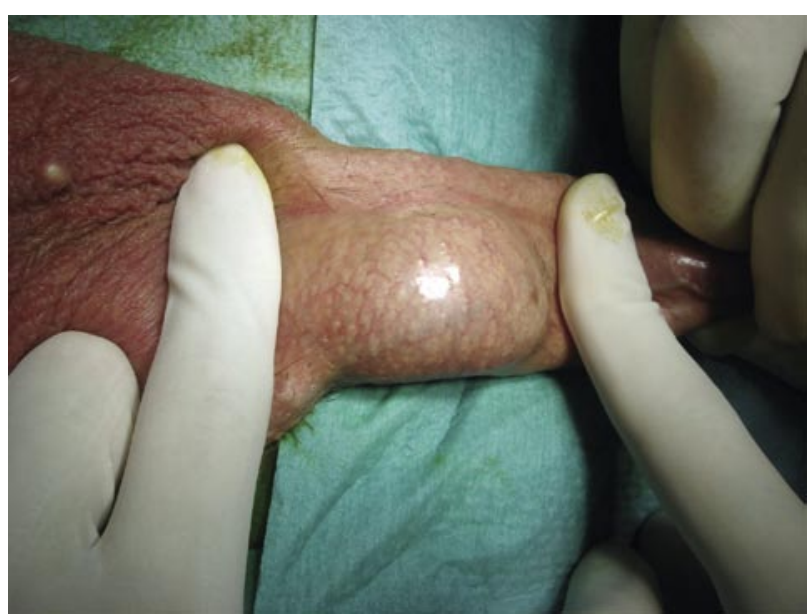

FIGURA 1. Divertículo relleno de suero salino. Maniobra previa a la cirugía. uno con lesiones mucosas que parecen secundarias a una alteración autoinmune sistémica que padece, estando en tratamiento actualmente con corticoides en espera de la evolución de su enfermedad, y otro que ha necesitado la revisión de uno de los extremos de la uretrostomía por reestenosis.

\section{DISCUSIÓN}

No es infrecuente encontrar una dilatación sacular o pseudodivertículo en un control radiológico tras una uretroplastia de sustitución, pudiendo dicho hallazgo ir acompañado de síntomas o permanecer asintomático $(2-4,7,13)$.

El mecanismo de producción es conocido, ya que se trata de una debilidad en la pared uretral que debe soportar la presión de la orina a su paso y que va cediendo precisamente en esa zona más débil que carece de soporte mecánico, a diferencia del resto de la uretra, configurando el divertículo. Este efecto puede ser más rápido si existe una estenosis más distal que va a incrementar la presión en esa zona (14).

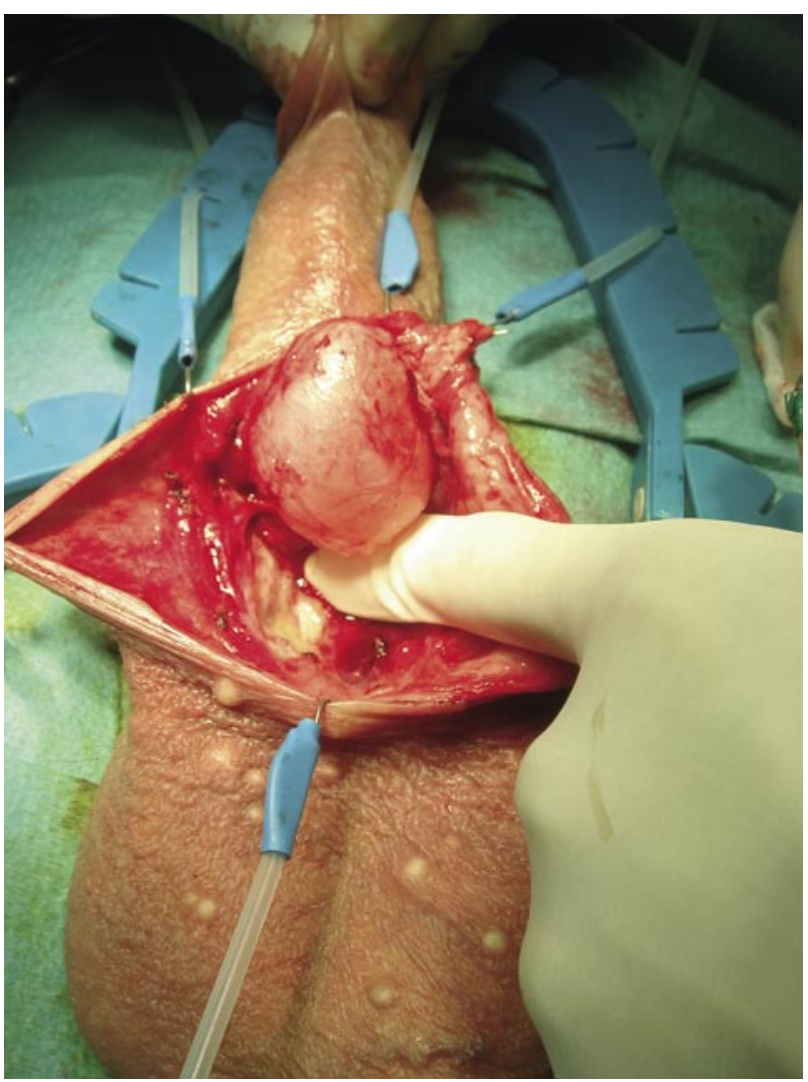

FIGURA 2. Liberación del divertículo uretral. 


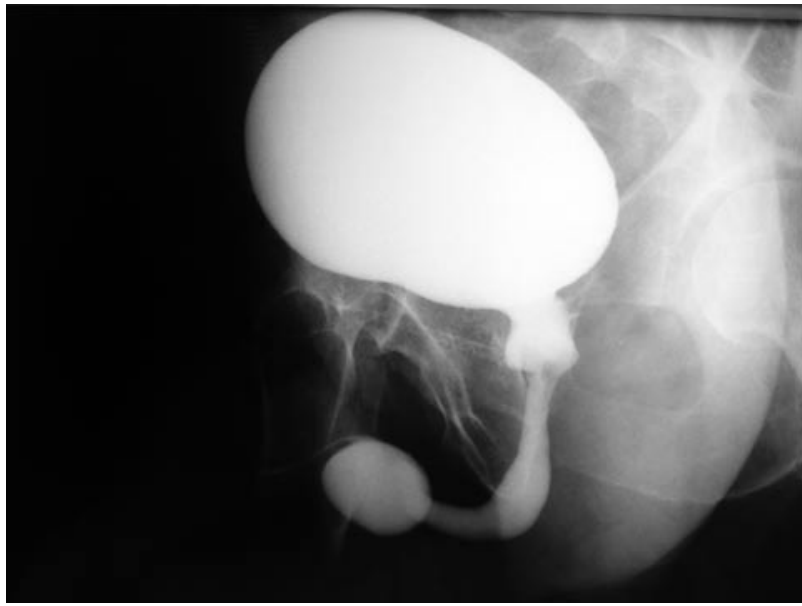

FIGURA 3. Cistouretrografía miccional.

Precisamente las técnicas que sitúan tanto injertos como colgajos en situación dorsal, lo que buscan es apoyar la neouretra en un soporte de mayor consistencia como es la albugínea de los cuerpos cavernosos que contrarreste la presión del flujo miccional $(1,2)$.

Desde el punto de vista clínico pueden ser sintomáticos y no sintomáticos. En el primer caso el paciente refiere un goteo tras finalizar la micción o tras la eyaculación, aparición de un bultoma blando y depresible en la zona operada (Figuras 1 y 2), dolor en ocasiones e incluso trastornos propios de una infección urinaria. Se han descrito casos de litiasis formadas en el interior de divertículos, con una sintomatología mucho más florida, o masas escrotales que simulaban tumoraciones $(5,6,10)$.

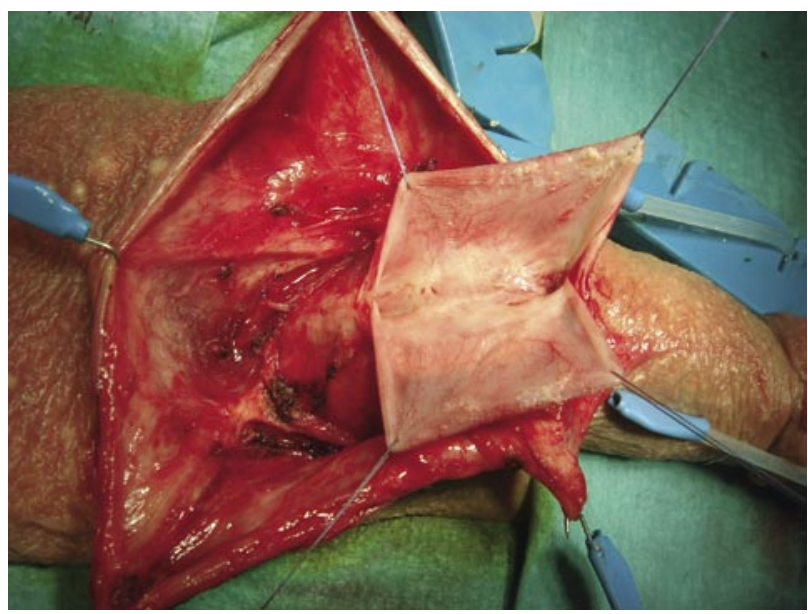

FIGURA 4. Divertículo abierto mostrando los dos orificios proximal y distal.
Para el diagnóstico es muy útil la cistouretrografía retrógrada y miccional, pues si sólo se realiza en sentido retrógrado puede quedar enmascarado el divertículo (Figura 3). También la ecografía uretral puede aportar datos al diagnóstico. La uretroscopia si no existe estenosis nos aportará datos del aspecto macroscópico de la mucosa y del contenido del divertículo.

El llevar a cabo el tratamiento va a depender en primera instancia de que existan o no síntomas, ya que ante un paciente asintomático puede considerarse una opción la abstención quirúrgica, con la recomendación de "exprimir" manualmente la uretra para completar su vaciado. En caso de que aparezcan trastornos severos, infecciones, dolor, litiasis o un goteo que resulte muy molesto para el sujeto, optaremos por el tratamiento quirúrgico, para lo cual disponemos de varias opciones.

\section{En uretra peneana}

- Si existe una estenosis distal asociada, corta, en uno de los extremos de la anastómosis y los síntomas son de predominio obstructivo, una uretrotomía interna puede ser la solución.

- Uretroplastia en un tiempo resecando el epitelio sobrante y conservando todo el tejido subepitelial que nos servirá para cubrir la sutura de la mucosa. (Figuras 4 y 5 ).

- Uretrostomía o marsupialización del divertículo si observamos signos inflamatorios en la mucosa, como erosiones, esfacelos, material purulento, etc. dejando el cierre definitivo para un segundo tiempo.

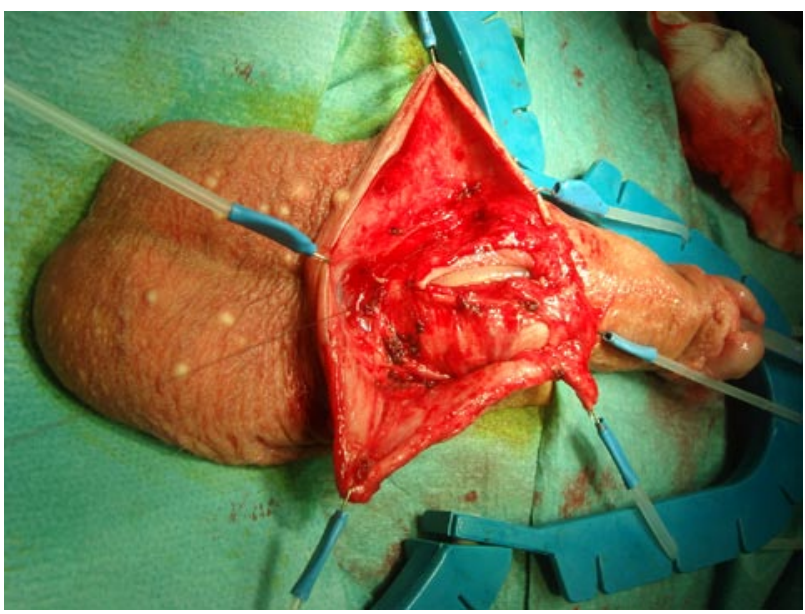

FIGURA 5. Cierre de la mucosa uretral sobre sonda vesical, tras la exéresis del tejido sobrante. 
- Resecar la mucosa sobrante, dejando una placa uretral y colocando un parche de mucosa bucal en su línea media con la técnica conocida como "snodgraft", de modo que ampliamos la luz uretral para en un segundo tiempo completar el cierre.

\section{En uretra bulbar}

- Uretrotomía interna si con ello solucionamos el problema obstructivo.

- Resección del tejido sobrante con vistas a reconstrucción en un tiempo, completando con una espongioplastia que servirá de soporte mecánico.

- Exéresis del divertículo y uretroplastia término-terminal combinada con injerto de mucosa oral, preferiblemente en situación dorsal.

Estas son algunas posibilidades de cirugía para utilizar en función de cada caso y de la experiencia del cirujano.

Al hacer referencia a divertículos o saculaciones nos estamos refiriendo a una complicación que no es infrecuente cuando se practica de forma habitual la cirugía de la uretra, y por ello autores como Barbagli, Mundy o Gil Vernet entre otros, maestros en las técnicas de la uretroplastia, han descrito modificaciones, variaciones o trucos para tratar de evitar su aparición $(2,8,9)$.

Así, en la uretra peneana, si vamos a realizar un colgajo tipo Orandi, una variación de la técnica consiste en rotar la uretra $180^{\circ}$ y suturar el colgajo, de forma que cuando aquella vuelva a su posición, éste quede en situación dorsal sobre la albugínea de los cavernosos.

Si optamos por un injerto libre también podemos colocarlo en posición dorsal, y si se sitúa en posición ventral no debemos olvidar la delgadez del tejido esponjoso, por lo que es aconsejable superponer a la sutura el dartos adyacente o en su defecto un colgajo de dicha fascia desplazado desde el escroto.

En la uretra bulbar los injertos libres podemos situarlos en posición dorsal según la técnica descrita por Barbagli o si estan en situación ventral realizar una buena espongioplastia. Puede resultar útil recubrir la sutura con gel de fibrina.

En el caso de los colgajos es aconsejable no sobredimensionar la neouretra y en caso de no realizar la espongioplastia recubrir la sutura con fascia de dartos.

\section{CONCLUSIONES}

La formación de pseudodivertículos uretrales - saculaciones, tras una cirugía de ampliación uretral por estenosis, es una consecuencia de la falta de apoyo o soporte mecánico del colgajo o injerto utilizado para reconstruir la uretra estenótica. Podemos tratar de evitar su aparición mediante técnicas que ayudan a soportar la presión del flujo miccional en una porción de uretra más debilitada por la cirugía, bien sea utilizando el plano de los cuerpos cavernosos o bien, en el caso de la posición ventral, superponiendo a la neouretra planos de refuerzo como el tejido esponjoso, la fascia de dartos.

Si diagnosticamos un divertículo uretral tras la cirugía optaremos por la abstención si no provoca síntomas o bien dispondremos de varias técnicas de acuerdo con la experiencia de cada cirujano.

\section{BIBLIOGRAFIAA y LECTURAS RECOMENDADAS ( ${ }^{*}$ lectura de interés $y^{* *}$ lectura fundamental)}

1. ANDRICH, D.E.; LEACH, C.J.; MUNDY, A.R.: "The Barbagli procedure gives the best results for patch urethroplasty of the bulbar urethra". BJU Int., 88: 385, 2001.

**2. BARBAGLI, G.; SELLI, C.; TOSTO, A. y cols.: "Dorsal free graft urethroplasty". J. Urol., 155: 123, 1996.

3. BARBAGLI, G.; PALMINTERI, E.; GUASÓN, G. y cols.: "Bulbar urethroplasty using bucal mucosa grafts placed on the ventral, dorsal or lateral surface of the uretra: are results affected by the surgical technique?". J. Urol., 174: 955, 2005.

*4. BHANDARI, M.; DUBEY, D.; VERMA, B.S.: "Dorsal or ventral placement of the preputial/penile skin flap for anterior urethral strictures: does it make a difference?". BJU Int., 88: 39, 2001.

5. BRIGMAN, J.A.; DETURE, F.A.: "Giant urethral diverticulum after free full thickness skin graft urethroplasty". J. Urol., 121: 523, 1979.

6. CREW, J.P.; NARGUND, V.; FELLOWS, G.J.: "Symptomatic urethral hair ball and diverticulum complicating island flap urethroplasty". J. Urol. Nephr., 30: 231, 1996.

**7. DE DIEGO, E.; MARTIN, B.; HERNANDEZ, R. y cols.: "Divertículos de uretra en el varón. Nuestra experiencia en 10 años". Arch. Esp. Urol., 51: 859, 1998.

8. GIL VERNET, J.M.; ARANGO, O.; GIL VERNET, A. y cols.: "A new biaxial epilated scrotal flap for reconstructive urethral surgery". J. Urol., 158: 412, 1997. 
9. GREENWELL, T.J.; VENN, S.N.; MUNDY, A.R.: "Cambio de práctica en la uretroplastia anterior". BJU Int., 83: 631, 1999.

10. JANG, T.L.; BLUNT, L.; YAP, R.L. y cols.: "Large urethral diverticulum presenting as a scrotal mass: urethral reconstruction with ventral onlay bucal mucosa”. J. Urol., 171: 351, 2004.

11. MARTINEZ, J.; LIEDANA, J.M.; RONCALES, A. y cols.: "Vigencia actual de la uretroplastia en dos tiempos". Actas Urol. Esp., 16: 549, 1992.

12. MELEKOS, M.D.; VERONIKIS, D.K.; SIAMPLIS, D. y cols.: "Diverticulum of the male urethra with a giant stone and multiple calculi". Urol. Int., 44: 184, 1989.

*13. MOREY, A.F.; DUCKETT, C.P.; McANINCH, J.W.: "Failed anterior urethroplasty: guidelines for reconstruction". J. Urol., 158: 1383, 1997.

14. RADOJICIC, Z.I.; PEROVIC, S.V.; DJORDJEVIC, M.L.J. y cols.: "Pseudospongioplasty in the repair of a urethral diverticulum". BJU Int., 94: 126, 2004. 